\title{
Famílias Constituídas por Lésbicas, Gays e Bissexuais: Revisão Sistemática de Literatura
}

\author{
Aline Nogueira de Lira ${ }^{1}$ \\ Normanda Araujo de Morais \\ Programa de Pós-Graduação em Psicologia da Universidade de Fortaleza, \\ Fortaleza, CE, Brasil
}

\begin{abstract}
Resumo
Buscou-se caracterizar a produção científica acerca das famílias constituídas por lésbicas, gays e bissexuais, a partir de uma revisão sistemática da literatura nacional e internacional, entre o período de 2009 a 2013. A busca foi feita nas bases Scientific Electronic Library Online (SciELO), Periódicos Eletrônicos de Psicologia (PePSIC), Literatura Latino-Americana e do Caribe em Ciências da Saúde (LILACS), Index Psi, PsycINFO e PUBMED, sendo identificados e analisados 145 artigos após a consideração dos critérios de inclusão e exclusão. Prevaleceram os estudos internacionais, empíricos, transversais, quantitativos e com ênfase na parentalidade lésbica. A análise de conteúdo dos artigos gerou a elaboração de duas categorias e suas sub-categorias, quais sejam: (a) Parentalidade (expectativas, percepções e transição para a parentalidade; estratégias de acesso à parentalidade; dinâmica e funcionamentos das relações pais/mães e filhos; homofobia e; discussões teóricas e metodológicas sobre a parentalidade); e (b) Conjugalidade (legalização do casamento; dinâmica conjugal; rede de apoio; e homofobia). Esta revisão ofereceu um panorama atual e abrangente dos estudos, sintetizando-o, lançando um olhar crítico a esse cenário multifaceado, além de indicar possibilidades para futuras investigações.
\end{abstract}

Palavras-chave: Família, parentalidade, conjugalidade, homossexualidade, mesmo sexo.

\section{Families Headed by Lesbian, Gays and Bisexuais: Systematic Literature Review}

\begin{abstract}
The aim of the present study was to describe the scientific literature on families constituted by lesbian, gay and bisexual individuals, through a systematic review of national and international studies published between 2009 and 2013. The following databases were searched: Scientific Electronic Library Online (SciELO), Periódicos Eletrônicos de Psicologia (PePSIC), Literatura Latino-Americana e do Caribe em Ciências da Saúde (LILACS), Index Psi, PsyINFO and PUBMED. After the application of inclusion and exclusion criteria, 145 articles were selected for review. Most studies were international, empirical, cross-sectional and quantitative, with a focus on lesbian parenthood. Content analysis of the articles revealed two major categories with a series of subcategories each: (a) Parenting (expectations, perceptions and transition to parenting; routes to parenthood; family dynamics and functioning; homophobia; and theoretical and methodological issues in the study of parenting in sexual minorities); (b) Marriage (marriage legalization; marital dynamics; support network; and homophobia). This review provides an updated and extensive discussion of the research into families headed by sexual minorities. Major findings are summarized and critically evaluated, and suggestions for future research are described.
\end{abstract}

Keywords: Family, parenting, marriage, homosexuality, same sex.

Endereço para correspondência: Rua Maria Teixeira Joca, 526, Casa 6, Centro, Eusébio, CE, Brasil 61760-000.

Fone: (85) 8726-2118. E-mail: aline.lira09@hotmail.com 


\section{Familias Compuestas por Lesbianas, Gays y Bisexuales: Revisión Sistemática de Literatura}

\section{Resumen}

Se ha buscado caracterizar la producción científica acerca de las familias compuestas por lesbianas, gays y bisexuales a partir de una revisión sistemática de la literatura nacional e internacional, en el período 2009-2013. La búsqueda se realizó en las bases Scientific Electronic Library Online (SciELO), Periódicos Eletrônicos de Psicologia (PePSIC), Literatura Latino-Americana e do Caribe em Ciências da Saúde (LILACS), Index Psi, PsycINFO y PUBMED. Se identificaron y se analizaron 145 artículos tras el examen de los criterios de inclusión y exclusión. Se han prevalecido los estudios internacionales, empíricos, transversales, cuantitativos y con énfasis en la parentalidad lesbiana. El análisis de contenido de los artículos ha generado dos categorías y sus subcategorías, a ver: (a) Parentalidad (expectativas, percepciones y transición para la parentalidad; estrategias de acceso a la parentalidad; dinámica y operación de las relaciones padres/madres e hijos; homofobia y; discusiones teóricas y metodológicas sobre la parentalidad); e (b) Conyugalidad (legalización del matrimonio; la dinámica de la pareja; redes de apoyo; y homofobia). Esta revisión ofreció un panorama largo y actual de los estudios, de forma a sintetizarlos con una visión crítica acerca del escenario múltiple, para allá de indicar posibilidades para futuras investigaciones.

Palabras clave: Familia, parentalidad, conyugalidad, homosexualidad, mismo sexo.

Os estudos sobre as famílias chefiadas por lésbicas, gays e bissexuais (LGB) ${ }^{2}$ têm florescido em número e objetivos nos últimos anos no mundo ocidental. As pesquisas sobre esta população começaram a ganhar destaque especialmente partir da década de 1970. Nessa época, tinham como objetivo analisar a saúde mental de mães lésbicas e o impacto potencialmente negativo quanto ao desenvolvimento psicossexual dos filhos frente à orientação sexual homossexual (Johnson, 2012). Os estudos da última década, porém, têm avançado nos processos e dinâmicas familiares, especialmente das mulheres lésbicas, registrando não só as dificuldades destas famílias, mas, sobretudo, considerando os pontos fortes destas experiências (Goldberg \& Gartrell, 2014).

É consenso na literatura que as famílias constituídas pela população LGB ainda sofrem discriminação, nos mais variados contextos -

A sigla LGB foi utilizada para referir-se, especificamente, aos estudos compostos por participantes lésbicas, gays e bissexuais, e que não incluem a categoria trans (travesti, transexual). A sigla LGBT será utilizada para descrever a "comunidade LGBT" de forma geral ou mesmo para indicar estudos específicos que incluem todos os subgrupos LGBT. sociais, sistema de saúde, sistema legal, nas escolas (Power et al., 2010). No entanto, um grande corpo de literatura científica acumulado ao longo de mais de 40 anos, oferece evidências sobre o bem-estar de crianças em famílias constituídas por casais do mesmo sexo, registrando que estes filhos não sofrem prejuízos em seu desenvolvimento psicossocial por causa da orientação sexual de seus pais (Goldberg \& Gartrell, 2014). Os resultados das últimas revisões de literatura realizadas sobre a parentalidade entre casais do mesmo sexo sobretudo com lésbicas, gays e bissexuais - revelam que, aspectos como a pobreza, depressão parental, divórcio, abuso de substâncias parental, violência doméstica são indicadores de fatores de risco para o desenvolvimento sociopsicológico infantil e adulto, mas a orientação sexual dos pais não está entre eles (Biblarz \& Stacey, 2010; Cecílio, Scorsolini-Comin, \& Santos, 2013; Goldberg \& Gartrell, 2014).

Sobre a conjugalidade entre casais do mesmo sexo, pesquisas empíricas demonstram que em vários aspectos, este subsistema familiar apresenta questões semelhantes às parcerias conjugais heterossexuais (Henderson, Lehavot, \& Simoni, 2009). Os amantes do mesmo sexo 
também formam laços emocionais profundos e comprometidos, além de enfrentarem problemas semelhantes em relação à intimidade, amor, lealdade e estabilidade da relação amorosa. No entanto, esses casais continuam a ser estigmatizados como resultado de sua condição de minoria sexual (Frost \& Meyer, 2009; Lomando, Wagner, \& Gonçalves, 2011). As experiências de homofobia ostensiva, ocultação da orientação sexual e homofobia internalizada - fatores estressores das minorias sexuais (Meyer, 2003), acabam por afetar negativamente a vida desses casais, diminuindo os níveis de satisfação conjugal, de intimidade e ainda afetando o bem-estar e a saúde mental desta população (Henderson et al., 2009).

Mesmo com o crescente corpo de estudos científicos sobre o tema "famílias e homossexualidade" e ainda o florescimento dos debates sociais e legais em relação a esta temática, vários mitos continuam a rondar o imaginário social acerca da vida dessas famílias, o que acaba por alimentar o preconceito homofóbico, por exemplo: o receio de que a orientação dos pais/mães possa prejudicar o desenvolvimento psicossocial dos filhos e que estes filhos se identifiquem como gays/lésbicas, ou ainda, associações entre a homossexualidade e a promiscuidade (Farias, 2010). Frente a esse cenário marcado pelo preconceito é que se justifica a importância da Psicologia avançar na produção de literatura sobre as famílias lideradas pela população LGB, discutindo e visibilizando as questões intrínsecas a esses arranjos familiares.

O presente estudo buscou, portanto, caracterizar a produção científica acerca das famílias constituídas por lésbicas, gays e bissexuais, a partir de uma revisão sistemática da literatura nacional e internacional, entre o período de 2009 a 2013. Ofereceu-se um panorama geral e atual das produções científicas, destacando-se os avanços, as lacunas e sinalizando áreas-chave que as pesquisas futuras devem responder. Além disso, realizou-se uma caracterização ampla da literatura, contemplando tanto as questões parentais, como as conjugais, uma vez que as famílias também se constituem pelo par conjugal, sem filhos. Ampliou-se ainda geograficamente o raio de abrangência das revisões de literatura - que, em sua maioria, referem-se à literatura norte-americana e europeia - incluindo artigos da América Latina e do Brasil, ao mesmo tempo em que se buscou sistematizar um amplo corpo de conhecimentos já produzido sobre o tema.

\section{Método}

Trata-se de uma revisão sistemática de literatura, com o objetivo de delinear a produção científica sobre as famílias constituídas pela população LGB.

\section{Procedimento}

Para nortear e sistematizar a execução da revisão da literatura seguiu-se oito etapas (Costa \& Zolowski, 2014):

1. Delimitação da questão norteadora;

2. Escolha das bases de dados;

3. Definição dos descritores para a busca;

4. Armazenamento dos resultados;

5. Seleção dos artigos pelo resumo, de acordo com os critérios de inclusão e exclusão;

6. Obtençãodosdados dos artigos selecionados;

7. Avaliação dos artigos; e

8. Síntese e interpretação dos dados.

\section{Coleta de Dados}

Elegeu-se as seguintes bases de dados para realizar a busca: Scientific Electronic Library Online (SciELO), Periódicos Eletrônicos de Psicologia (PePSIC), Literatura Latino-Americana e do Caribe em Ciências da Saúde (LILACS), Index Psi, PsycINFO e PUBMED. Em português foram utilizados os seguintes descritores de busca com operadores booleanos: "(homossexual $O R$ lésbica $O R$ gay $O R$ homoparental $O R$ lgbt) $A N D$ (famílias $O R$ parentalidade $O R$ conjugalidade $O R$ casamento $O R$ maternidade $O R$ paternidade)". Já em inglês foram empregados os termos em inglês a seguir: "(homosexual OR lesbian $O R$ gay $O R$ lgbt OR same-sex) AND (families OR parenting OR parenthood OR mother OR father)". Em francês aplicou-se o descritor, "homoparentalité". A busca nas bases de dados foi realizada nos meses de junho e julho de 2014. Dessa forma, estabeleceu-se o período entre 
2009 e 2013 como referência, com o intuito de mapear as publicações recentes, dada as transformações legais, jurídicas, sociais e acadêmicas em relação ao tema "família e homossexualidade" nos últimos cinco anos e ao crescente número de publicações. Além disso, estudos de revisão, especialmente sobre a parentalidade LGB, foram publicados em momentos anteriores, oferecendo um olhar retrospectivo do panorama da produção científica, o que justifica ainda mais a importância desse recorte temporal (e.g. Biblarz \& Stacey, 2010; Cecílio et al., 2013; Vecho \& Schneider, 2005). Este período foi considerado satisfatório para apontar as principais tendências e resultados do tema pesquisado.

Destacam-se os seguintes critérios de inclusão: artigos indexados; redigidos nos idiomas português, inglês, espanhol e francês; artigos teóricos e empíricos; disponíveis em sua versão completa; publicados entre os anos de 2009 a 2013; e realizada com seres humanos. Posteriormente foram analisados os resumos (abstracts), sendo que foram considerados os critérios de exclusão listados a seguir: artigos duplicados; capítulos de livro; notícias; documentos técnicos; comentários; outras revisões de literatura (sistemática e narrativa); dissertações e teses; publicados fora do período entre 2009 a 2013; e estudos cujo objetivo tratava do tema da homossexualidade, mas não se relacionava às famílias.

Optou-se por não incluir as revisões sistemática de literatura no corpus de análise, em coerência com o objetivo deste estudo que é caracterizar a produção científica acerca das famílias constituídas pela população $\mathrm{LGB}$, em termos de objetivos, métodos, temas e resultados. Outras revisões serviram para situar os achados já publicados com os encontrados no presente trabalho (e.g. Biblarz \& Stacey, 2010; Cecílio et al., 2013; Goldberg \& Gartrell, 2014; Santos, Scorsolini-Comin, \& Santos, 2013).

Após uma primeira seleção, realizada pelo exame dos resumos de acordo com os critérios de inclusão e exclusão, os artigos foram recuperados por completo e, nesse momento, submetidos à avaliação de dois juízes independentes uma estudante da graduação e uma estudante da pós-graduação, ambas integrantes do grupo de pesquisa coordenado pela primeira autora desse artigo sobre famílias constituídas por casais do mesmo sexo. Em seguida, realizou-se o Teste de Relevância (Azevedo, 2010). As exclusões foram esclarecidas pelos juízes e as divergências resolvidas em consenso entre eles. Por fim, os estudos selecionados foram lidos na íntegra e a extração dos dados foi armazenada em planilhas do Excel.

\section{Análise dos Dados}

Para a síntese e interpretação dos resultados, foram realizadas dois tipos de análises: a primeira, de natureza quantitativa, na qual se buscou traçar o perfil da produção científica, considerando questões como: ano de publicação; nacionalidade das instituições dos primeiros autores; periódicos; idioma; tipo de estudo; método; delineamento; instrumentos; perfil dos participantes e principais arranjos familiares. Na segunda análise, de natureza qualitativa, organizou-se e fez-se uma síntese dos conteúdos temáticos (Bardin, 1977/1979) e somente a posteriori mapearam-se duas grandes categorias de análise - parentalidade e conjugalidade - e suas subcategorias. A identificação das (sub) categorias foi feita a partir das etapas a seguir: (a) a pré-análise - organizou-se as temáticas através de leitura flutuante dos artigos revisados, formulando-se hipóteses, objetivos e elaborando indicadores de análise; (b) a exploração dos estudos - os dados foram explorados e codificados a partir de unidades de registro; e, (c) o tratamento dos resultados e interpretação - foi realizada a categorização das temáticas a partir de classificações dos dados, de acordo com suas semelhanças e por diferenciação. Após a definição das categorias temáticas, os dados foram apresentados e discutidos a partir de relações feitas com a literatura específica da referida área de estudo.

\section{Resultados e Discussão}

Identificou-se mediante a busca inicial nas bases de dados um total de 4.869 estudos (PUBMED: 4.589; PsycINFO: 159; SciELO: 42; PePSIC: 23; LILACS: 40; IndexPsi: 16). Após a leitura dos resumos (abstracts) aplicou-se os 
critérios de inclusão e exclusão, chegando aos seguintes dados: artigos duplicados $(n=48)$; artigos fora do período entre 2009 a 2013 (n $=3.698)$; artigos com não humanos $(n=101)$; abstracts não acessíveis nas bases de dados ( $n$ $=77)$; literatura cinza $(n=87)$ estudos que não problematizavam o tema "família e homossexualidade" $(n=563)$.

Após esta primeira seleção, chegou-se a um número de 295 estudos, e mesmo depois de utilizar o serviço de comutação bibliográfica e de entrar em contato com alguns autores, só foi possível recuperar o texto completo de 289 artigos, representando significativamente, $97,96 \%$ da amostra.

Estes artigos foram encaminhados aos juízes e submetidos ao teste de relevância (Azevedo, 2010). Dentre os principais motivos de exclusão após a análise dos juízes destacam-se: artigos que não incluíam nos seus objetivos questões familiares, mas apenas tratavam de temas ligados à identidade LGBT (por exemplo, suicídio em jovens homossexuais) ou ainda artigos que investigavam as relações conjugais, mas a população era composta somente por casais solteiros sem filhos; e ainda, pela falta de clareza entre os objetivos do estudos e seu alcance. Por fim, foram incluídos 145 estudos que compuseram o corpo de análise deste trabalho, sendo: PUBMED ( $n=$ 109); PsycINFO ( $n=17)$; SciELO $(n=10)$; PePSIC $(n=7)$; LILACS $(n=1)$ e IndexPsi $(n=1)$. As referências completas dos estudos incluídos nesta revisão sistemática, estão destacadas com asterisco $(*)$.

\section{Perfil Quantitativo das Produções Científicas}

A Tabela 1 apresenta a caracterização quantitativa dos artigos analisados. A maioria dos estudos foi redigida no idioma inglês $(n=125)$, seguido por 16 artigos em português. Quanto às instituições de origem dos autores dos artigos, a maioria, é proveniente dos Estados Unidos $(n=$ 77), seguida pelo Brasil $(n=15)$ e Reino Unido $(n=11)$. Quanto ao período das publicações, no ano de 2013 foram publicados 31 artigos. Em 2012 foram 31 artigos, em $2011(n=28), 2010$ $(n=30)$ e $2009(n=25)$.
Acerca das questões metodológicas foram recuperados 122 artigos empíricos e 23 estudos teóricos. Nos estudos empíricos percebeu-se que a maioria é de delineamento transversal ( $n$ = 110), embora 12 estudos sejam longitudinais. Quanto às pesquisas nacionais, localizaram-se 7 artigos teóricos e 8 artigos empíricos (todos com delineamento transversal), sinalizando um crescimento nas investigações empíricas nos últimos anos. Predominaram as abordagens quantitativas $(n=62)$, sendo qualitativo $(n=53)$. Apenas 7 artigos utilizaram a abordagem de multimétodos. Sobre os instrumentos de coleta de dados, nos estudos empíricos quantitativos, sobressaiu o uso de escalas, questionários padronizados e inquéritos auto-aplicados de estilo psicométrico. Para o exame dos estudos quantitativos predominaram as análises de qui-quadrado, análises multivariadas e análises de regressão. Dois estudos desenvolveram e validaram escalas acerca da parentalidade: por exemplo, Scale Beliefs about Children's Adjustment on Same-sex Families - SBCASSF (Frias-Navarro \& Monterde-i-Bort, 2012). Ressalta-se a importância de validar mais escalas com população LGB, uma vez que muitos dos instrumentos de coleta foram desenvolvidos para a população heterossexual. Já nas abordagens qualitativas, utilizou-se, predominantemente, entrevistas (estruturadas e semi-estruturadas). Para a análise dos dados, no geral, realizou-se análise de conteúdo por temas.

O tamanho das amostras/participantes dos estudos variou de 1 a 6.864 pessoas. Pesquisas com lésbicas (solteiras, casadas, divorciadas) predominaram nesta revisão, especialmente, quando enfocavam a parentalidade $(n=33)$, seguido por gays $(n=23)$. Apesar de não ter sido utilizado o descritor transgênero na busca nas bases de dados, três estudos incluíram esta população nos dados empíricos, discutindo-os no contexto da homossexualidade (Chapman et al., 2012; Knoble \& Linville, 2012; Zanghellini, 2010). Sobre as pesquisas com filhos, foram localizados 19 estudos, sendo crianças $(n=6)$, adolescentes e adultos jovens $(n=11)$, adultos $(n=1)$ e idosos $(n=1)$. É notável o crescimento de estudos que abordam a vida dos filhos da população LGB, embora ainda seja muito pequeno o núme- 
ro pesquisas, principalmente, com filhos adultos. Foram localizados, ainda, estudos com amostras compostas por adultos heterossexuais - consumidores, cristãos, estudantes universitários, profissionais da saúde e da área escolar $(n=17)$.

Dois grandes temas podem caracterizar a produção científica das famílias constituídas por pela população LGB: estudos sobre a parentalidade $(n=92)$ e sobre a conjugalidade $(n=53)$. $\mathrm{O}$ predomínio, sobretudo, de pesquisas sobre a parentalidade, pode estar relacionado às preocupações com os efeitos da orientação sexual dos pais nos níveis de ajustamento psicossocial das crianças (Biblarz \& Stacey, 2010; Santos et al., 2013). Sobre os principais arranjos familiares investigados na literatura nos últimos cinco anos, referem-se notadamente à reprodução assistida e adoção. Foram localizados dois estudos com enfoque na coparentalidade (Tarnovski, 2011, 2013). É possível indicar que estes resultados estão relacionados à crescente mudança nas questões legais, jurídicas e tecnológica nos últimos anos, tendo um impacto nas definições e arranjos familiares.

Tabela 1

Caracterização Quantitativa dos Estudos Recuperados $(n=145)$

\begin{tabular}{|c|c|c|}
\hline $\begin{array}{c}\text { Categorias } \\
\text { Quantitativas }\end{array}$ & $\begin{array}{l}\text { Amostra } \\
\text { (Geral) }\end{array}$ & $\begin{array}{l}\text { Amostra } \\
\text { (Brasil) }\end{array}$ \\
\hline Ano & $\begin{array}{l}2013(n=31) \\
2012(n=31) \\
2011(n=28) \\
2010(n=30) \\
2009(n=25)\end{array}$ & $\begin{array}{l}2013(n=2) \\
2012(n=2) \\
2011(n=5) \\
2010(n=2) \\
2009(n=4)\end{array}$ \\
\hline $\begin{array}{l}\text { Nacionalidade } \\
\text { de origem do } \\
\text { primeiro autor/ } \\
\text { Estados no } \\
\text { Brasil }\end{array}$ & $\begin{array}{c}\text { EUA }(n=77) \\
\text { Brasil }(n=15) \\
\text { Reino Unido }(n=11) \\
\text { Austrália }(n=10) \\
\text { Canadá }(n=7) \\
\text { Portugal }(n=4) \\
\text { Holanda, Espanha e Israel (cada país, } n=3) \\
\text { Suécia, Noruega e Bélgica }(n=2) \\
\text { Dinamarca, China, Colômbia, França, Hungria e Taiwan }(n=1)\end{array}$ & $\begin{array}{c}\text { São Paulo }(n=5) \\
\text { Rio de Janeiro }(n=4) \\
\text { Mato Grosso e Recife }(n=2) \\
\text { Minas Gerais, Paraíba, } \\
\text { Porto Alegre }(n=1)\end{array}$ \\
\hline Tipo de estudo & $\begin{array}{l}\text { Empírico }(n=122) \\
\text { Teórico }(n=23)\end{array}$ & $\begin{array}{l}\text { Empírico }(n=8) \\
\text { Teórico }(n=7)\end{array}$ \\
\hline Delineamento & $\begin{array}{l}\text { Transversal }(n=110) \\
\text { Longitudinal }(n=12)\end{array}$ & $\begin{array}{l}\text { Transversal }(n=9) \\
\text { Longitudinal }(n=0)\end{array}$ \\
\hline $\begin{array}{l}\text { Método de } \\
\text { análise }\end{array}$ & $\begin{array}{l}\text { Quantitativo }(n=62) \\
\text { Qualitativo }(n=53) \\
\text { Multimétodos }(n=7)\end{array}$ & $\begin{array}{l}\text { Quantitativo }(n=1) \\
\text { Qualitativo }(n=8)\end{array}$ \\
\hline $\begin{array}{l}\text { Amostra/ } \\
\text { Participantes }\end{array}$ & $\begin{array}{c}\text { Gays }(n=23) \\
\text { Lésbicas }(n=33) \\
\text { Filhos }(n=19) \\
\text { Gays e Lésbicas }(n=22) \\
\text { Gays, lésbicas, bissexuais e heterossexuais }(n=11) \\
\text { Transgêneros }(n=3) \\
\text { Profissionais de saúde, educadores, universitários }(n=17)\end{array}$ & $\begin{array}{l}\text { Gays }(n=3) \\
\text { Lésbicas }(n=2) \\
\text { Filhos }(n=1) \\
\text { Gays e Lésbicas }(n=2) \\
\text { Profissionais de saúde, } \\
\text { educadores, } \\
\text { universitários }(n=1)\end{array}$ \\
\hline Instrumento & $\begin{array}{l}\text { Questionários e escalas }(n=44) \\
\text { Entrevistas }(n=51)\end{array}$ & $\begin{array}{c}\text { Questionários e escalas }(n=1) \\
\text { Entrevistas }(n=8)\end{array}$ \\
\hline Tema & $\begin{array}{l}\text { Parentalidade }(n=92) \\
\text { Conjugalidade }(n=53)\end{array}$ & $\begin{array}{l}\text { Parentalidade }(n=12) \\
\text { Conjugalidade }(n=3)\end{array}$ \\
\hline
\end{tabular}




\section{Caracterização Qualitativa dos Estudos}

A análise qualitativa dos estudos teve como critério a classificação dos artigos que abordavam o conjunto de temas relacionados às vivências familiares da população LGB - parentais e conjugais - destacando os seus principais resultados. Ofereceu-se, ainda, uma perspectiva crítica do material revisto, evidenciando os pontos fortes, bem como os limites e direções para futuras investigações.

Parentalidade em Lésbicas, Gays e Bissexuais. A partir da categoria parentalidade, chegou-se aos seguintes subtemas: (a) expectativas, percepções e transição quanto à parentalidade ( $n$ = 13); (b) estratégias de acesso à parentalidade ( $n=23)$; (c) dinâmicas e funcionamentos das relações pais/mães-filhos $(n=22)$; (d) vivências de preconceito e o impacto da homofobia na vida das famílias homoparentais $(n=25)$; (e) discussões teóricas e metodológicas sobre a parentalidade $(n=9)$. A Tabela 2 apresenta exemplos de referências para cada subcategoria, assim como a frequência de artigos encontrados em cada uma delas.

Sobre as expectativas, percepções e transição quanto à parentalidade, quando comparados com os casais heterossexuais, os estudos indicam que gays e lésbicas mostraram-se menos propensos a expressarem os desejos de parentalidade - sobretudo pelos desafios legais e preocupações quanto ao desenvolvimento psicossocial dos filhos - no entanto, aprovaram o valor da paternidade/maternidade tão fortemente como fez participantes heterossexuais, além de se perceberam habilitados para o exercício parental (e.g. Goldberg \& Smith, 2009; Riskind \& Patterson, 2010). De modo geral, os estudos indicam que os principais obstáculos para gays e sobretudo para as mulheres lésbicas decidirem ter filhos são: os desafios legais e jurídicos (Ryan, 2013), as preocupações quanto à dificuldade de engravidar pela falta de apoio social durante o processo de concepção (Yager, Brennan, Steele, Epstein, \& Ross, 2010) e as preocupações sobre o próprio exercício da parentalidade, especialmente quanto ao desenvolvimento psicossocial dos filhos (Riskind \& Patterson, 2010; Riskind, Patterson, \& Nosek, 2013). No entanto, lésbicas e gays mostraram-se confiantes em superar as dificuldades - legais, econômicas e sociais - para a realização da parentalidade, principalmente quando se trata da adoção, mais do que através da parentalidade biológica (Riskind et al., 2013). As mulheres lésbicas mostram-se mais propensas a realizarem adoções inter-raciais, quando comparadas com os casais heterossexuais, mesmo que estejam conscientes dos desafios que envolvem a sua condição (Goldberg, 2009).

Ainda é limitado o número de estudos que têm enfocado a questão da transição da parentalidade em famílias formadas pela população LGB, um momento tão significativo para a vida de qualquer família. No geral, os estudos desta revisão sugerem que os pais/mães gays e lésbicas apresentam questões semelhantes à transição aos pais heterossexuais, por exemplo: a saúde mental e a qualidade conjugal podem diminuir com a chegada dos filhos (Goldberg \& Smith, 2011); a paternidade, porém, pode aumentar a dedicação do cônjuge ao seu relacionamento primário e reduzir o tempo e a sua energia para a manutenção de relacionamento amoroso, fato geralmente relacionado à diminuição da satisfação sexual (Goldberg \& Smith, 2009).

Apesar dos avanços, as pesquisas relacionadas às expectativas, percepções e transição para a parentalidade, como por exemplo, com inclusão da população gay nas amostras investigadas, algumas questões ainda precisam ser respondidas, a saber: quais as expectativas de pais/mães sobre a parentalidade de pessoas solteiras e de casais? E como é a experiência de transição de parentalidade quando uma da díade do casal já tem filhos? Como as minorias sexuais atravessam a parentalidade em termos de recursos financeiros e educacionais? E como estas famílias têm buscado apoio para aliviar o estresse nesse momento de transição de suas vidas? (Goldberg \& Gartrell, 2014).

Sobre as principais estratégias de acesso à parentalidade destacam-se a parentalidade planejada, através da reprodução assistida (doação de espermas e barriga de aluguel) e da adoção, sobretudo nos estudos publicados a partir do final da década de 1990 e início dos anos 2000. Tal fato representa uma mudança significativa 
no modo como as famílias lideradas pela população LGB têm seus filhos, já que anteriormente prevaleciam as investigações acerca da experiência de pais e mães que tiveram seus filhos em relações heterossexuais, anteriores às vivências amorosas homossexuais, conforme destacado pelo estudo de revisão de Biblarz e Stacey (2010).

Um dos arranjos familiares que mais ganhou visibilidade na literatura nos últimos anos, especialmente, pelo avanço e acesso às tecnologias reprodutivas e as mudanças legislativas recentes, foram os estudos sobre a experiência de mulheres lésbicas que se utilizam da reprodução assistida para ter filhos (e.g. Grover, Shmorgun, Moskovtsev, Baratz, \& Librach, 2013; Moás \& Correa, 2010), com enfoque nos aspectos éticos, legais e de intimidade do casal quanto à decisão sobre o uso do esperma do doador (conhecido ou desconhecido), e as implicações desta escolha para a conjugalidade e o desenvolvimento psicossocial dos filhos (e.g. Bos \& Gartrell, 2010a). A eleição por doadores desconhecidos é decorrente, principalmente, na tentativa de evitar um terceiro envolvido na vida do filho e do casal e o receio de que o doador possa requerer a custódia dos filhos (e.g. Goldberg \& Allen, 2013). Já a escolha pelo doador conhecido pode ser mobilizada pela ideia de que seus filhos merecem ter acesso ao seu patrimônio genético ou mesmo para que suas crianças tenham pelo menos um modelo masculino (e.g. Goldberg \& Allen, 2013). Em conjunto, a literatura ressalta que o acesso à informação sobre as origens de doadores de uma criança é importante para alguns pais e tem consequências potencialmente positivas para os filhos, aumentando o senso de identidade destes (e.g. Freeman, Jadva, Kramer, \& Golombok, 2009). Ressalta-se o crescimento de estudos que examinam a vida dos filhos adolescentes e jovens adultos de mães lésbicas que foram concebidos através da reprodução assistida e como eles foram criados e conheceram o significado e construíram suas relações com os doadores conhecidos (e.g. Goldberg \& Allen, 2013).

Sobre a barriga de aluguel/solidária, os estudos registram que esta tem sido uma estratégia utilizada por gays - em parcerias conjugais ou solteiros - para construírem suas famílias (e.g. Greenfeld \& Seli, 2011; Smith, Willmott,
Trowse, \& White, 2013). Além disso, estudos apontam a estreita relação entre o crescente número de homens gays que procuram tratamento de fertilidade e a legalização do casamento (e.g. Grover et al., 2013). Uma das perguntas ainda sem reposta neste cenário é como as crianças nascidas através de um acordo de sub-rogação se sentem em relação as suas mães de aluguel?

É importante destacar que tanto os estudos que envolvem a doação de espermas (no caso das lésbicas), como a barriga de aluguel/solidária (com os gays) são voltados, quase que exclusivamente, para homens e mulheres que têm elevadas condições socioeconômicas. É necessário, portanto, a realização de novas pesquisas que explorem os demarcadores econômicos, raciais, de classe e de gênero envolvidos nessa tomada de decisão, para ampliar a representatividade das amostras e então chegar a novos achados da literatura.

Sobre a adoção, as evidências mais notáveis encontradas nesta revisão apontam que os laços emocionais entre pais/mães e filhos adotivos, os resultados de ajustamento psicossocial das crianças adotadas na primeira infância e as habilidades parentais durante a transição para a parentalidade adotiva, parecem não variar com base no gênero ou orientação sexual dos pais/mães (e.g. Goldberg \& Smith, 2013). A parentalidade adotiva de pais/mães gays e lésbicas se constroem no cotidiano, inclusive para alguns pais desde o primeiro momento que iniciam a relação com a crianças adotadas (Amazonas, Veríssimo, \& Lourenço, 2013). Outro dado relevante e que corrobora o que estudos anteriores vêm apontando, é sobre a presença do alto estresse parental de pais/mães LGB adotivos - frente ao contexto marcado pela discriminação social, legal, familiar (e.g. Tornello, Farr, \& Patterson, 2011). No entanto, um importante avanço nas investigações é sobre como os pais e mães homossexuais respondem à discriminação no processo de adoção e as estratégias encontradas por eles para enfrentarem o preconceito e adotar os seus filhos (Bos \& Gartrell, 2010b).

Sobre as dinâmicas e funcionamento dos pais/mães e seus filhos, as produções científicas mais recentes continuam a ratificar o que a literatura já vem dizendo ao longo das décadas 
anteriores (Biblarz \& Stacey, 2010; Goldberg \& Gartrell, 2014): os filhos de famílias homoparentais parecem não sofrer prejuízos em seu desenvolvimento sócio-cognitivo-psico-sexual em virtude da orientação sexual dos pais (e.g. Bos \& Gartrell, 2010a; Farr, Forssell, \& Patterson, 2010). No geral, as pesquisas sobre o ajustamento psicossocial centraram-se, principalmente, em fazer análises comparativas com as famílias heterossexuais, demonstrando, de modo geral, que os mesmos fatores que estão ligados ao desenvolvimento positivo dos filhos de pais heterossexuais, também estão relacionados aos filhos de famílias homoparentais: qualidade relacional entre os membros da família (proximidade e boa comunicação entre pais e filhos, satisfação conjugal); a força na segurança de apego; competências parentais; os índices de saúde mental dos pais/mães (por exemplo, ausência de depressão parental); ausência de violência doméstica; não abuso de substâncias psicoativas (e.g. Gartrell \& Bos, 2010). Sobre essa categoria, um importante avanço metodológico e teórico dos últimos anos refere-se à realização de pesquisas longitudinais centradas nos processos de desenvolvimento e não somente nos resultados, de modo a considerar a influência de múltiplas interações (sociais, culturais, legais, situações de vida, familiares) e variações de fatores (protetivos e de risco) que se intercruzam e colaboram para o ajustamento psicossocial dos filhos (e.g. Gartrell, Bos, Peyser, Deck, \& Rodas, 2012). Além disso, as pesquisas têm incluído a percepção que os filhos (crianças e adolescentes e adultos jovens) têm a respeito de suas famílias, assinalando uma diferença significativa, relativa ao paradigma inicial das investigações, o qual se centrava exclusivamente na percepção dos pais/mães acerca de seus filhos (e.g. Gartrell, Bos, \& Goldberg, 2011).

Acerca da influência dos pais/mães na orientação sexual dos seus filhos, apesar da literatura não ter encontrado diferenças relevantes entre filhos de pai/mães do mesmo sexo e filhos de pais heterossexuais, em sua auto-identificação como gays/lésbicas (Tasker \& Golombok, 1997), nesta revisão os estudos de Schumm (2010) sugerem que haja uma tendência em haver transferência intergeracional da orientação sexual, especial- mente para as mães lésbicas e crianças do sexo feminino. As investigações científicas longitudinais realizadas por Gartrell et al. (2011), também indicaram que as filhas adolescentes de mães lésbicas são mais propensas a se envolverem em comportamento do mesmo sexo e se identificar como bissexual. $\mathrm{O}$ que a literatura não explica é se as filhas de lésbicas são mais propensas a vivenciarem relações do mesmo sexo influenciadas pela orientação sexual de suas mães, ou pela possibilidade de que os adolescentes com mães lésbicas podem demonstrar noções mais expansivas e menos categóricas acerca da sexualidade (Cohen \& Kuvalanka, 2011).

Sobre o desempenho da parentalidade dos gays, lésbicas e bissexuais - incluindo as competências e a qualidade das relações entre pais e filhos - foram identificadas, sobretudo, muitas semelhanças entre as famílias homoparentais e as famílias compostas por pares heterossexuais, evidenciando que a orientação sexual dos pais/ mães não está relacionada às consequências ou aos resultados do exercício parental (Farr \& Patterson, 2013). Quando existem diferenças, contudo, estas parecem favorecer aos casais do mesmo sexo - especialmente composto por mulheres lésbicas - em várias competências, como: casais de lésbicas mostraram-se mais igualitárias na divisão de tarefas domésticas, na interação e cuidados com os filhos, nos processos de tomada de decisão e na participação em atividades com os filhos, quando comparadas aos casais heterossexuais (e.g. Farr \& Patterson, 2013). Ressalta-se, porém, que é preciso ter cuidado com essa concepção de igualitarismo presente nas famílias lésbicas, negligenciando as suas diferenças e ainda acreditando que o compartilhamento equitativo entre os cônjuges é a melhor opção para todas as famílias (Goldberg \& Gartrell, 2014). Alguns casais podem não se encaixar nesse modelo, e assumir papéis diferenciados e específicos de acordo, por exemplo, com a facilidade para a execução das tarefas ou mesmo de acordo com o contexto familiar em que os filhos foram concebidos (e.g. Martinez \& Barbieri, 2011).

Ainda sobre a parentalidade, considerável número de pesquisas registram que as vivências de preconceito e o impacto da homofobia na vida 
das famílias homoparentais como resultado de sua orientação sexual dos pais/mães (Bos \& Gartrell, 2010b; Rodriguez \& Paiva, 2009). Gays, lésbicas, transgêneros e seus filhos podem sofrer discriminação ou disparidade, por exemplo, nos serviços de saúde - no período pré e pós-natal ou no acesso ao seguro de saúde privado entre os filhos de casais do mesmo sexo, na assistência social, na educação e nos sistemas jurídicos (e.g. Chapman et al., 2012). Teorias de efeitos estressores sobre minorias sexuais foram analisadas e continuam a indicar categoricamente que o impacto da homofobia (externa e/ou interna) pode repercutir negativamente na saúde física e mental da população LGB (Meyer, 2003), ou mesmo no bem-estar psicológico dos filhos (e.g. Bos \& Gartrell, 2010 a), ao lidarem com os contextos legais e sociais, que nem sempre dão suporte às relações conjugais e parentais. No entanto, com base nos achados da literatura desta revisão, sugere-se que efeitos adversos da homofobia podem ser amortecidos pelo fortalecimento da rede de apoio, seja no sistema intrafamiliar (na interação pais-filhos, no sistema conjugal), na família de origem, no trabalho, com os amigos, e ainda no âmbito legal e institucional (e.g. Bos \& Gartrell, 2010b; Lomando et al., 2011).

Ainda sobre o preconceito, as pesquisas apontam que o consenso público - expresso por profissionais de saúde e da área escolar, por estudantes universitários, por cristãos, pela população em geral heterossexual - é esmagador de que crianças criadas por famílias heterossexuais se desenvolvem com mais sucesso do que os filhos de gays e lésbicas (e.g. Ferreira, Cadete, \& da Silva, 2012; Gato \& Fontaine, 2013). Os resultados desta revisão continuam a ser consistentes com estudos anteriores (e.g. Herek, 2000) em que as atitudes das pessoas eram mais negativas em relação à parentalidade gay do que as das lésbicas, sugerindo que o padrão das atitudes variam de acordo com o sexo das minorias sexuais (e.g. Costa et al., 2013).

Por fim, alguns artigos científicos abordam de forma abrangente discussões teóricas e metodológicas acerca das questões familiares da população LGBT. Resumidamente, estes estudos apontam reflexões teóricas sobre as novas formas de pensar a família, ultrapassando as identidades cristalizadas centradas no modelo heterossexista (e.g. Rodriguez \& Gomes, 2012; Vilhena, Souza, Uziel, Zamora, \& Novaes, 2011). Outros trabalhos validaram escalas sobre parentalidade (e.g. Frias-Navarro \& Monterde-i-Bort, 2012) e apresentaram panoramas dos projetos teóricos e metodológicos de estudos longitudinais (e.g. Power et al., 2010).

Conjugalidade em Lésbicas, Gays e Bissexuais. Quatro temas principais caracterizaram as produções científicas do período de 2009-2013 quanto à conjugalidade em casais do mesmo sexo: (a) legalização do casamento $(n=17)$; (b) dinâmica das relações conjugais $(n=19)$; (c) rede de apoio $(n=10)$ e; (d) homofobia e o seu impacto na vida dos cônjuges $(n=9)$. A Tabela 2 apresenta exemplos de referências para cada subcategoria.

As discussões em torno da legalização do casamento entre pessoas do mesmo sexo têm se concentrado, de modo geral, em justificar ou combater a ideia de que o status legal do casamento para esses casais pode enfraquecer a instituição familiar e que isto prejudicaria o desenvolvimento psicossocial dos seus possíveis filhos (e.g. Knauer, 2012). As evidências científicas, no entanto, sugerem que a oficialização jurídica do casamento pode oferecer efeitos positivos na promoção do bem-estar físico e psicológico, diminuindo as disparidades em saúde mental entre lésbicas, gays, bissexuais e heterossexuais e promovendo benefícios tangíveis às suas vidas (Wight, LeBlanc, \& Lee Badgett, 2013), o que pode colaborar para o exercício saudável da parentalidade. Após o reconhecimento legal do casamento, gays e lésbicas, por exemplo, diminuíram significativamente o uso de assistência médica e utilização dos serviços de saúde mental (Hatzenbuehler et al., 2012); houve também, redução dos índices de mortalidade entre gays e lésbicas, apesar dessas taxas de mortalidade ainda superarem as da população em geral (Frisch \& Brønnum-Hansen, 2009); e, por fim, gays e lésbicas revelaram menos estresse psicológico (menores índices de homofobia internalizada, de sintomas depressivos e estresse) e maior bem-estar psicológico (e.g. Riggle, Rostosky, \& Horne, 2010). De acordo com a análise dos artigos revi- 
sados, os efeitos potencialmente positivos com a legalização do casamento podem ocorrer em decorrência dos benefícios econômicos tangíveis; de um senso de estabilidade no relacionamento associado com o reconhecimento legal do compromisso matrimonial; dos efeitos positivos de intimidade e proximidade; bem como a partir de um maior apoio emocional e auto-estima conferido ao casamento (e.g. Wight et al., 2013). Futuras pesquisas precisam, entretanto, investigar de que forma o status de legalidade e ajustamento psicossocial dos cônjuges (uso de drogas, bem-estar subjetivo, satisfação de vida, dentre outras) estão relacionados. Além disso, o impacto desta legalização para a saúde física e psicológica dos filhos desses casais também merece atenção. Estudos longitudinais são indicados para esta temática.

Sobre a dinâmica das relações conjugais homossexuais, estudos demonstram que mudanças legais relacionadas ao casamento podem ter influenciado a maneira que as gerações mais jovens de casais do mesmo sexo conceituam e vivem seus relacionamentos. Evidencia-se, por exemplo, que acordos não monogâmicos têm diminuído entre estes casais, que aspiram ter relacionamentos estáveis, exclusivos e de longo prazo com os/as parceiros/as (Gotta et al., 2011). Ressalta-se, no entanto, que a literatura registra que as relações monogâmicas não estão diretamente associadas à satisfação sexual entre os cônjuges. Mas, a quebra dos acordos sexuais entre os parceiros (e.g. acordos de não envolvimento emocional, apenas de sexo casual) e as discrepâncias de vivências entre eles (e.g. um dos parceiros vive mais experiências extraconjugais do que o outro parceiro) podem afetar tanto a satisfação conjugal, como a satisfação sexual dos cônjuges (Mitchell, Harvey, Champeau, Moskowitz, \& Seal, 2012).

Os principais fatores associados aos níveis de satisfação conjugal encontrados nesta revisão foram: exteriorização da orientação sexual, respeito aos acordos sexuais entre os parceiros/ as, comunicação, coesão, funcionamento sexual e a rede de apoio social (e.g. Knoble \& Linville, 2012). Por sua vez, os altos níveis de satisfação conjugal podem ter efeitos positivos sobre o bem-estar físico e psicológico dos casais do mesmo sexo.

Apesar de escassas, no geral, as pesquisas sobre a satisfação sexual de casais de lésbicas apontaram um conjunto de fatores preditores homólogos, tanto na vida de mulheres lésbicas, bissexuais, como nas mulheres heterossexuais, a saber: satisfação conjugal, rede de apoio social (inclusive com a legalização do casamento), funcionamento e prazer sexual e ausência de sintomotalogia depressiva (e.g. Henderson et al., 2009). Em lésbicas e bissexuais a homofobia internalizada, assim com a carência das condições socioeconômicas foram citadas como aspectos que influenciam negativamente os índices de sua satisfação sexual (Henderson et al., 2009).

No âmbito das relações domésticas, companheiras lésbicas relataram viver relações mais igualitárias na divisão de finanças e na comunicação, e apoio entre as cônjuges mais equitativos quando comparados aos casais heterossexuais. Já os gays relataram menos igualdade de comunicação, menor compartilhamento de apoio, e menos equidade na tomada de decisão do que os homens heterossexuais (Gotta et al., 2011).

Evidências científicas nesta revisão informam que a rede de apoio (social, jurídica, familiar) é importante fator preditivo para a satisfação conjugal e relaciona-se diretamente ao bem-estar dos cônjuges, podendo amortecer o impacto negativo do estresse advindo, especialmente do preconceito (e.g. Lomando et al., 2011). Observou-se a importância da rede de apoio em situações, por exemplo, em que os cônjuges podiam contar efetivamente com a ajuda e apoio socioemocional da família de origem; ou mesmo diante de situações de doenças, como o câncer de mama; ou mesmo para ajudar gays em situações de luto, ao perderem seus parceiros por causas não relacionadas ao HIV (e.g. Hornjatkevyc $\&$ Alderson, 2011). O apoio social pode ser, portanto, um fator de proteção importante diante das adversidades que os casais do mesmo sexo possam vivenciar, protegendo-os de uma variedade de fatores negativos advindos da discriminação homofóbica.

Os estudos analisados na presente revisão avançam sobre a compreensão da homofobia 
e o seu impacto na vida dos cônjuges do mesmo sexo, bem como nas estratégias encontradas por eles para superar os efeitos da discriminação (Frost, 2011). A homofobia tanto pode ser vivenciada externamente, através da discriminação ostensiva, como pode ser internalizada pelas minorias sexuais (Meyer, 2003). Os estudos desta revisão registram uma correlação positiva entre a homofobia internalizada (na medida em que as minorias sexuais internalizam atitudes negativas sobre a homossexualidade), e efeitos na saúde mental em homens e mulheres homossexuais (Frost \& Meyer, 2009). Verificou-se, po- rém, que alguns casais de gays e lésbicas vivenciaram o estigma, como uma oportunidade para (re) definir noções de compromisso e legitimidade relacional, além de ser uma oportunidade para aproximá-los aos seus parceiros e fortalecer o vínculo dentro de seus relacionamentos (Frost, 2011). Os resultados desta revisão sugerem, portanto, que lidar com o estigma sexual - interno e externo - é fundamental para o bem-estar psicológico e engajamento social dos amantes do mesmo sexo. Novas pesquisas precisam avançar nos estudos sobre a homofobia internalizada, pois ela pode ser um indicador chave da saúde mental (Frost \& Meyer, 2009).

Tabela 2

Principais Subcategorias e Exemplos de Referências nos Estudos Recuperados sobre a Parentalidade e Conjugalidade

\section{Temáticas sobre a Parentalidade LGB}

1. Estudos sobre expectativas, percepções e transição para a parentalidade $(n=13)$

2. Principais formas de acesso à parentalidade (reprodução assistida, adoção e coparentalidade; $n=23$ )

3. Dinâmicas e funcionamento das vivências dos filhos, dos pais/mães e a relação entre eles (ajustamento psicossocial dos filhos, desempenho dos papéis parentais, orientação sexual dos pais/mães e os filhos; $n=22$ )

4. Vivências de Preconceito (o impacto da homofobia na vida das famílias, atitudes e crenças em relação a parentalidade LGB; $n=25$ )

5. Discussões teóricas e metodológicas sobre a parentalidade (discussões teóricas gerais sobre as famílias homoparentais; métodos de pesquisa e validação de escalas na parentalidade; $n=9$ )

\section{Exemplos de Referências}

Goldberg \& Smith, 2009, 2011; Naziri

\& Feld-Elzon, 2012; Riskind \& Patterson, 2010

Amazonas et al., 2013; Bos \& Gartrell, 2010b;

Freeman et al., 2009; Goldberg \& Allen, 2013;

Greenfeld \& Seli, 2011; Moás \& Correa, 2010

Bos \& Gartrell, 2010a; Cohen \& Kuvalanka, 2011; Farr et al., 2010; Gartrell et al., 2011; Martinez \& Barbieri, 2011; Schumm, 2010

Bos \& Gartrell, 2010b; Chapman et al., 2012; Ferreira et al., 2012; Gato \& Fontaine, 2013; Rodriguez \& Paiva, 2009

Frias-Navarro \& Monterde-i-Bort, 2012; Vilhena et al., 2011

\section{Temáticas sobre a Conjugalidade LGB}

1. Debates acerca da legalização do casamento homossexual e implicações destas mudanças na saúde física e mental dos cônjuges $(n=17)$

2. Dinâmica do relacionamento entre os pares do mesmo sexo (satisfação conjugal, satisfação sexual, tarefas domésticas; $n=19$ )

3. Rede de apoio social aos casais homoconjugais $(n=10)$

4. Homofobia e o seu impacto na vida dos casais $(n=9)$

\section{Exemplos de Referências}

Hatzenbuehler et al., 2012; Riggle et al., 2010; Wight et al., 2013

Gotta et al., 2011; Henderson et al., 2009; Knoble \& Linville, 2012; Mitchell et al., 2012

Hornjatkevyc \& Alderson, 2011;

Lomando et al., 2011

Frost, 2011; Frost \& Meyer, 2009 


\section{Considerações Finais}

Este estudo teve como objetivo apresentar um panorama atual da produção científica acerca das famílias constituídas pela população LGB, no cenário nacional e internacional. Houve um florescimento em número e alcance da produção científica nos últimos cinco anos (2009-2013) em torno desta temática. No geral, predominam os estudos internacionais, empíricos, transversais, quantitativos e que utilizam as entrevistas (em pesquisas qualitativas) e escalas e questionários padronizados (em estudos quantitativos), como principais técnicas de coletas de dados. No cenário brasileiro, verificou-se que apesar das pesquisas estarem em franca expansão, os estudos ainda são muito teóricos e quando empíricos, em sua maioria, são qualitativos, com populações pouco representativas.

Sobre os participantes, prevalece o número de estudos que investigam a maternidade lésbica, apesar do crescimento de estudos com gays e também com os filhos das minorias sexuais. Os estudos sobre a população bissexual e de transgênero, bem como os seus filhos (especialmente adultos), encontram-se ainda mais limitados e merecem atenção futura. Sobre os principais temas de investigação científica, predominaram os estudos acerca da parentalidade em detrimento da conjugalidade. Houve um crescimento no número de estudos que investigavam os arranjos familiares construídos através da parentalidade planejada: reprodução assistida e adoção.

No cenário da parentalidade, evidencia-se, com base na racionalidade científica dos últimos anos, que os níveis de ajustamento psicossocial e bem-estar psicológico de crianças e adolescentes parecem não estar relacionados à orientação sexual dos pais/mães. A qualidade da dinâmica relacional familiar entre pais/mães e seus filhos, são os principais preditores para avaliar as competências parentais. No entanto, a homofobia (externa e/ou interna) pode ser um indicador chave nos índices de saúde física e mental dos pais/mães ou mesmo no bem-estar psicológico dos filhos (Bos \& Gartrell, 2010a; Goldberg \& Smith, 2009).
Quanto à conjugalidade, acompanhando as mudanças legais em torno do casamento homossexual, as produções científicas têm dedicado atenção aos efeitos positivos que a legalização do casamento pode trazer para a vida dos casais do mesmo sexo, estando diretamente associada ao bem-estar físico e psicológico dos cônjuges. Além disso, a literatura aponta que casais do mesmo sexo aspiram viver relacionamentos monogâmicos, duradouros e apresentam questões e dilemas semelhantes às parcerias heterossexuais (Frost, 2011; Gotta et al., 2011; Henderson et al., 2009). A homofobia, e o seu impacto na vida dos casais do mesmo sexo parece exercer influência na saúde mental da população LGB, tendo consequência nos níveis de bem-estar psicológico e engajamento social.

Apesar dos notáveis avanços em termos do tamanho e da representatividade das amostras (participantes) e considerações sobre os processos de desenvolvimento - especialmente através dos estudos longitudinais - uma variedade de problemas metodológicos caracterizam as pesquisas sobre a população LGB. A maioria dos estudos é composta por amostras pouco representativas - realizados predominantemente com adultos gays e lésbicas, brancos, que têm condição socioeconômica mais elevada e que são residentes de regiões metropolitanas. Estes estudos, portanto, não podem ser generalizados à população bissexual e trans (transgêneros, transexuais e travestis), à população de idosos, às minorias sexuais negras, de baixa renda e residentes de áreas rurais que podem viver em dupla condição de minorias. Além disso, predominam os estudos com corte transversal, portanto, os efeitos causais não podem ser assumidos nesses estudos.

Além disso, vários temas específicos têm recebido pouca ou nenhuma atenção na literatura dos últimos anos, por exemplo: vivências de luto (dos parceiros íntimos ou mesmo dos filhos); divórcio e recasamentos; violência pelo parceiro íntimo em casais de lésbicas (Hardesty, Oswald, Khaw, \& Fonseca, 2009); com filhos adultos; tentativas inexitosas de reprodução assistida; funcionamento da família quando um ou ambos 
os pais LGB tem HIV / AIDS ou uma doença crônica; impacto da legalização do casamento na vida dos filhos; como os pais dialogam com os filhos sobre a sexualidade; e estudos com populações de casais constituídos por pessoas mais velhas. Por fim, sugere-se a realização de estudos que considerem, de forma integrada, a participação de todos os membros da família.

Uma das principais limitações desta revisão refere-se à eleição de descritores que deram ênfase a gays e lésbicas, não incluindo os transgêneros, travestis e transexuais. Talvez por isso, evidenciou-se um pequeno número de estudos sobre esta população. Por outro lado, como os descritores em sua maioria faziam referência à homossexualidade, parece que os estudos não incluem essa população nesta categoria.

Frente à natureza exploratória do presente estudo e a crescente expansão das publicações científicas em torno das famílias formadas por lésbicas, gays e bissexuais, ressalta-se que a mais notável contribuição desta revisão foi oferecer um panorama atual e abrangente dos estudos, sintetizando-o, lançando um olhar crítico a esse cenário complexo e multifaceado, além de indicar possibilidades para futuras investigações.

\section{Referências}

*Amazonas, M. C. L. D. A., Veríssimo, H. V., \& Lourenço, G. O. (2013). A adoção de crianças por gays. Psicologia \& Sociedade, 25(3), 631641. doi:10.1590/S0102-71822013000300017

Azevedo, R. S. (2010). Sobrecarga do cuidador informal da pessoa idosa frágil: Uma revisão sistemática (Dissertação de mestrado não publicada, Universidade Federal de Minas Gerais, Belo Horizonte, MG, Brasil).

Bardin, L. (1979). Análise de conteúdo (L. Reto \& A. Pinheiro, Trads.). São Paulo, SP: Edições 70. (Original publicado em 1977)

Biblarz, T. J., \& Stacey, J. (2010). How does the gender of parents matter? Journal of Marriage and Family, 72, 3-22. doi:10.1111/j.17413737.2009.00678.x

*Bos, H. M. W., \& Gartrell, N. K. (2010a). Adolescents of the US National Longitudinal Lesbian Family Study: The impact of having a known or an unknown donor on the stability of psycholo- gical adjustment. Human Reproduction, 26(3), 630-637. doi:10.1093/humrep/deq359

*Bos, H. M. W., \& Gartrell, N. K. (2010b). Adolescents of the USA National Longitudinal Lesbian Family Study: Can family characteristics counteract the negative effects of stigmatization? Family Process, 49(4), 559-572. doi:10.1111/j.1545-5300.2010.01340.x

*Cecílio, M. S., Scorsolini-Comin, F., \& Santos, M. A. D. (2013). Scientific production on adoption by gay couples in Brazilian context. Estudos de Psicologia (Natal), 18(3), 507-516. doi:10.1590/ S1413-294X2013000300011

*Chapman, R., Wardrop, J., Freeman, P., Zappia, T., Watkins, R., \& Shields, L. (2012). A descriptive study of the experiences of lesbian, gay and transgender parents accessing health services for their children. Journal of Clinical Nursing, 21(7 - 8), 1128-1135. doi:10.1111/ j.1365-2702.2011.03939.x

*Cohen, R., \& Kuvalanka, K. A. (2011). Sexual socialization in lesbian-parent families: An exploratory analysis. American Journal of Orthopsychiatry, 81(2), 293. doi:10.1111/ j.1939-0025.2011.01098.x

Costa, A. B., \& Zolowski, A. P. C. (2014). Como escrever um artigo de revisão sistemática. In S. H. Koller, M. C. P. P. Couto, \& J. Von Hohendorff (Eds.), Manual de Produção Científica (pp. 5570). Porto Alegre, RS: Penso.

*Costa, P. A., Caldeira, S., Fernandes, I., Rita, C., Pereira, H., \& Leal, I. (2013). Atitudes da população portuguesa em relação à Homoparentalidade. Psicologia: Reflexão e Crítica, 26(4), 790798. doi:10.1590/S0102-79722013000400020

*Farias, M. O. (2010). Mitos atribuídos às pessoas homossexuais e o preconceito em relação à conjugalidade homossexual e a homoparentalidade. Revista de Psicologia da UNESP, 9(1), 104115. Recuperado em http://186.217.160.122/ revpsico/index.php/revista/article/downlo$\mathrm{ad} / 169 / 211$

*Farr, R. H., Forssell, S., \& Patterson, C. J. (2010). Parenting and child development in adoptive families: Does parental sexual orientation matter? Applied Developmental Science, 10, 164-178. doi:10.1080/10888691.2010.500958

*Farr, R. H., \& Patterson, C. J. (2013). Coparenting among lesbian, gay, and heterosexual couples: Associations with adopted children's outco- 
mes. Child Development, 84(4), 1226-1240. doi:10.1111/cdev.12046

*Ferreira, S. P. A., Cadete, V. G., \& da Silva, D. B. (2012). Os sentidos e os significados produzidos pela escola em relação à família homoparental: Um estudo de caso. Interação em Psicologia, 16(1). doi:10.5380/psi.v16i1.13947

*Freeman, T., Jadva, V., Kramer, W., \& Golombok, S. (2009). Gamete donation: Parents' experiences of searching for their child's donor siblings and donor. Human Reproduction, 24(3), $505-$ 516. doi:10.1093/humrep/den469

*Frias-Navarro, D., \& Monterde-i-Bort, H. (2012). A scale on beliefs about children's adjustment in same-sex families: Reliability and validity. Journal of Homosexuality, 59(9), 1273-1288. doi:10. 1080/00918369.2012.720505

*Frisch, M., \& Brønnum-Hansen, H. (2009). Mortality among men and women in same-sex marriage: A National Cohort Study of 8333 Danes. American Journal of Public Health, 99(1), 133. doi:10.2105/AJPH.2008.133801

*Frost, D. M. (2011). Stigma and intimacy in same-sex relationships: A narrative approach. Journal of Family Psychology, 25(1), 1. doi:10.1037/ a0022374

*Frost, D. M., \& Meyer, I. H. (2009). Internalized homophobia and relationship quality among lesbians, gay men, and bisexuals. Journal of Counseling Psychology, 56, 97-109.

*Gartrell, N. K., \&, Bos, H. M. W. (2010). The US National Longitudinal Lesbian Family Study: Psychological adjustment of 17-year-old adolescents. Pediatrics, 126, 1-9. doi:10.1542/ peds.2009-3153

*Gartrell, N. K., Bos, H. M., \& Goldberg, N. G. (2011). Adolescents of the US National Longitudinal Lesbian Family Study: Sexual orientation, sexual behavior, and sexual risk exposure. Archives of Sexual Behavior, 40(6), 1199-1209. doi:10.1007/s10508-010-9692-2

*Gartrell, N. K., Bos, H. M. W., Peyser, H., Deck, A., \& Rodas, C. (2012). Adolescents with lesbian mothers describe their own lives. Journal of Homosexuality, 59, 1211-1229. doi:10.1080/ 00918369.2012 .720499

*Gato, J., \& Fontaine, A. M. (2013). Anticipation of the sexual and gender development of children adopted by same-sex couples. International
Journal of Psychology, 48(3), 244-253. doi:10. 1080/00207594.2011.645484

*Goldberg, A. E. (2009). Lesbian and heterosexual preadoptive couples' openness to transracial adoption. American Journal of Orthopsychiatry, 79(1), 103. doi:10.1037/a0015354

*Goldberg, A. E., \& Allen, K. R. (2013). Donor, dad, or...? Young adults with lesbian parents' experiences with known donors. Family Process, 52(2), 338-350. doi:10.1111/famp.12029

Goldberg, A. E., \& Gartrell, N. K. (2014). LGBparent families: The current state of the research and directions for the future. Advances in Child Development and Behavior, 46, 57-88. doi:10.1016/B978-0-12-800285-8.00003-0

*Goldberg, A. E., \& Smith, J. Z. (2009). Perceived parenting skill across the transition to adoptive parenthood among lesbian, gay, and heterosexual couples. Journal of Family Psychology, 23(6), 861. doi:10.1037/a0017009

*Goldberg, A. E., \& Smith, J. Z. (2011). Stigma, social context, and mental health: Lesbian and gay couples across the transition to adoptive parenthood. Journal of Counseling Psychology, 58(1), 139. doi:10.1037/a0021684

*Goldberg, A. E., \& Smith, J. Z. (2013). Predictors of psychological adjustment among early placed adopted children with lesbian, gay, and heterosexual parents. Journal of Family Psychology, 27, 431-442. doi:10.1037/a0032911

*Gotta, G., Green, R. J., Rothblum, E., Solomon, S., Balsam, K., \& Schwartz, P. (2011). Heterosexual, lesbian, and gay male relationships: A comparison of couples in 1975 and 2000. Family Process, 50(3), 353-376. doi:10.1111/j.15455300.2011.01365.x

*Greenfeld, D. A., \& Seli, E. (2011). Gay men choosing parenthood through assisted reproduction: Medical and psychosocial considerations. Fertility and Sterility, 95(1), 225-229. doi:10.1016/j.fertnstert.2010.05.053

*Grover, S. A., Shmorgun, Z., Moskovtsev, S. I., Baratz, A., \& Librach, C. L. (2013). Assisted reproduction in a cohort of same-sex male couples and single men. Reproductive Biomedicine Online, 27(2), 217-221. doi:10.1016/j. rbmo.2013.05.003

*Hardesty, J. L., Oswald, R. F., Khaw, L., \& Fonseca, C. (2009). Lesbian/bisexual mothers 
and intimate partner violence: Help seeking in the context of social and legal vulnerability. Violence against Women, 17(1), 28-46. doi:10.1177/1077801209347636

*Hatzenbuehler, M. L., O’Cleirigh, C., Grasso, C., Mayer, K., Safren, S., \& Bradford, J. (2012). Effect of same-sex marriage laws on health care use and expenditures in sexual minority men: A quasi-natural experiment. American Journal of Public Health, 102(2), 285-291. doi:10.2105/ AJPH.2011.300382

*Henderson, A. W., Lehavot, K., \& Simoni, J. M. (2009). Ecological models of sexual satisfaction among lesbian/bisexual and heterosexual women. Archives of Sexual Behavior, 38(1), 5065. doi:10.1007/s10508-008-9384-3

Herek, G. M. (2000). Sexual prejudice and gender: Do homosexuals attitudes toward lesbian and gay men differ? Journal of Social Issues, 56, 251-266.

*Hornjatkevyc, N. L., \& Alderson, K. G. (2011). With and with out: The bereavement experiences of gay men who have lost a partner to non-AIDS-related causes. Death Studies, 35(9), 801-823. doi:10.1080/07481187.2011.553502

Johnson, S. M. (2012). Lesbian mothers and their children: The third wave. Journal of Lesbian Studies, 16(1), 45-53. doi:10.1080/10894160.2 011.557642

*Knauer, N. J. (2012). Legal consciousness and LGBT research: The role of the law in the everyday lives of LGBT individuals. Journal of Homosexuality, 59(5), 748-756. doi:10.1080/00 918369.2012 .673947

*Knoble, N. B., \& Linville, D. (2012). Outness and relationship satisfaction in same-gender couples. Journal of Marital and Family Therapy, 38(2), 330-339. doi:10.1111/j.17520606.2010.00206.x

*Lomando, E., Wagner, A., \& Gonçalves, J. (2011). Coesão, adaptabilidade e rede social no relacionamento conjugal homossexual. Psicologia: Teoria e Prática, 13(3), 96-109. Recuperado em http://pepsic.bvsalud.org/scielo.php?script=sci arttext\&pid $=$ S1516-36872011000300008

*Martinez, A. L. M., \& Barbieri, V. (2011). A experiência da maternidade em uma família homoafetiva feminina. Estudos de Psicologia (Campinas), 28(2), 175-185. doi:10.1590/ S0103-166X2011000200005
Meyer, I. H. (2003). Prejudice, social stress, and mental health in lesbian, gay, and bisexual populations: Conceptual issues and research evidence. Psychological Bulletin, 129, 674-697. doi:10.1037/0033-2909.129.5.674

*Mitchell, J. W., Harvey, S. M., Champeau, D., Moskowitz, D. A., \& Seal, D. W. (2012). Relationship factors associated with gay male couples' concordance on aspects of their sexual agreements: Establishment, type, and adherence. AIDS and Behavior, 16(6), 15601569. doi:10.1007/s10461-011-0064-2

*Moás, L. D. C., \& Correa, M. C. D. V. (2010). Filiação e tecnologias de reprodução assistida: Entre medicina e direito. Physis, 20(2), 591-607. doi:10.1590/S0103-73312010000200014

*Naziri, D., \& Feld-Elzon, E. (2012). Becoming a mother by "aid" within a lesbian couple: The issue of the third. The Psychoanalytic Quarterly, 81(3), 683-711. doi:10.1002/j.2167-4086.2012. tb00514.x

*Power, J. J., Perlesz, A., Schofield, M. J., Pitts, M. K., Brown, R., McNair, R., ...Bickerdike, A. (2010). Understanding resilience in same-sex parented families: The work, love, play study. $B M C P u$ blic Health, 10(1), 115. doi:10.1186/1471-245810-115

*Riggle, E. D., Rostosky, S. S., \& Horne, S. G. (2010). Psychological distress, well-being, and legal recognition in same-sex couple relationships. Journal of Family Psychology, 24(1), 82. doi:10.1037/a0017942

*Riskind, R. G., \& Patterson, C. J. (2010). Parenting intentions and desires among childless lesbian, gay, and heterosexual individuals. Journal of Family Psychology, 24(1), 78. doi:10.1037/ a0017941

*Riskind, R. G., Patterson, C. J., \& Nosek, B. A. (2013). Childless lesbian and gay adults' self-efficacy about achieving parenthood. Couple and Family Psychology: Research and Practice, 2(3), 222. doi:10.2139/ssrn.2095376

*Rodriguez, B. C., \& Gomes, I. C. (2012). Novas formas de parentalidade: Do modelo tradicional à homoparentalidade. Boletim de Psicologia, 62(136), 29-36. Recuperado em http:// pepsic.bvsalud.org/scielo.php?pid=S0006$-59432012000100004 \&$ script $=$ sci arttext

*Rodriguez, B. C., \& Paiva, M. L. D. S. C. (2009). Um estudo sobre o exercício da parentalida- 
de em contexto homoparental. Vínculo, 6(1), 13-25. Recuperado em http://pepsic.bvsalud.org/scielo.php?script $=$ sci arttext\&pid $=\mathrm{S} 1806-24902009000100003$

*Ryan, M. (2013). The gender of pregnancy: Masculine lesbians talk about reproduction. Journal of Lesbian Studies, 17(2), 119-133. doi:10.1080/10 894160.2012 .653766

Santos, Y. G. S., Scorsolini-Comin, F., \& Santos, M. A. (2013). Homoparentalidade masculina: Revisando a produção científica. Psicologia: Reflexão e Crítica, 26(3), 572-582. doi:10.1590/ S0102-79722013000300017

*Schumm, W. R. (2010). Children of homosexuals more apt to be homosexuals? A reply to Morrison and to Cameron based on an examination of multiple sources of data. Journal of Biosocial Science, 42(06), 721-742. doi:10.10017/ S0021932010000325

*Smith, M. K., Willmott, L., Trowse, P., \& White, B. P. (2013). Back to the future: Prohibiting surrogacy for singles, same-sex and shorter-term heterosexual couples in Queensland. Journal of Law and Medicine, 20(3), 638-654. Retrieved from http://www.ncbi.nlm.nih.gov/ pubmed/23600195

*Tarnovski, F. L. (2011). Les coparentalités entre gays et lesbiennes en France: le point de vue des pères. Vibrant: Virtual Brazilian Anthropology, 8(2), 140-163. doi:10.1590/S180943412011000200007

*Tarnovski, F. L. (2013). Parentalidade e gênero em famílias homoparentais francesas. Cadernos Pagu, 40, 67-93. doi:10.1590/S010483332013000100002

Tasker, F. L., \& Golombok, S. (1997). Growing up in a lesbian family: Effects on child development. London: Guilford Press.

*Tornello, S. L., Farr, R. H., \& Patterson, C. J. (2011). Predictors of parenting stress among gay adoptive fathers in the United States. Journal of Family Psychology, 25(4), 591. doi:10.1037/ a0024480
Vecho, O., \& Schneider, B. (2005). Homoparentalité et développement de l'enfant: bilan de trente ans de publications. La Psychiatrie de l'Enfant, 481, 271-328. doi:10.3917/psye.481.0271

*Vilhena, J. D., Souza, A. C. B. D., Uziel, A. P., Zamora, M. H., \& Novaes, J. V. (2011). Que família?: Provocações a partir da homoparentalidade. Revista Mal Estar $e$ Subjetividade, 11(4), 1639-1658. Recuperado em http://pepsic.bvsalud.org/scielo.php?script $=$ sci arttext\&pid $=$ S1518-61482011000400014

*Wight, R. G., LeBlanc, A. J., \& Lee Badgett, M. V. (2013). Same-sex legal marriage and psychological well-being: Findings from the California Health Interview Survey. American Journal of Public Health, 103(2), 339-346. doi:10.2105/ AJPH.2012.301113

*Yager, C., Brennan, D., Steele, L. S., Epstein, R., \& Ross, L. E. (2010). Challenges and mental health experiences of lesbian and bisexual women who are trying to conceive. Health \& Social Work, 35(3), 191-200. Retrieved from http:// www.ncbi.nlm.nih.gov/pubmed/20853646

*Zanghellini, A. (2010). Queer kinship practices in non-western contexts: French Polynesia's gender-variant parents and the law of $\mathrm{La}$ République. Journal of Law and Society, 37(4), 651-677. Retrieved from http://www.ncbi.nlm. nih.gov/pubmed/21125769
Recebido: 29/05/2015

$1^{a}$ revisão: $31 / 08 / 2015$ Aceite final: 10/09/2015 